\title{
Influence of Hydrogen Induced Thermal Pinch on Nanoparticle Formation in Arc Plasma*
}

\author{
by Junichi Noma ${ }^{* *}$, Hiroya Abe ${ }^{* * *}$, Makio Naito ${ }^{* * *}$, Kentaro Yamamoto***, Shinichi Tashiro ${ }^{* * *}$ and Manabu Tanaka ${ }^{* * *}$ \\ Fe nanoparticles were prepared by DC arc plasma method under $\mathrm{Ar}, \mathrm{Ar}-50 \% \mathrm{H}_{2}$ or $\mathrm{H}_{2}$ flow. The arc plasma was well constricted due to \\ thermal pinch effect when $\mathrm{Ar}-50 \% \mathrm{H}_{2}$ or $\mathrm{H}_{2}$ was used, and the generation ration of the Fe nanoparticles was significantly enhanced with the \\ pinched arc. Our numerical simulation indicated that the Fe anode can be efficiently heated (close to boiling point of $\mathrm{Fe}$ ) due to the thermal \\ pinch effect, and thereby the generation ratio can be increased. Since the thermal pinch effect enhanced the arc flow velocity as well, the size \\ of the resulting Fe nanoparticles was also influenced.
}

Key Words: DC arc, Thermal pinch effect, Nanoparticles, Generation rate, Hydrogen, Input power

\section{Introduction}

DC arc plasma can result in a localized high energy density, giving a very high temperature with capable of melting and vaporizing metals, and simultaneously induced plasma jet can rapidly quench vaporized metals. Thus, the arc plasma can be act as self flow reactor for producing nanoparticles. Because it is a route to prepare high-purity nanoparticles with high productivity at low cost, the arc plasma technique has attracted great interest 1-3).

Ohno and Uda developed an effective method for production of metallic nanoparticles by using hydrogen gas ${ }^{4,5}$. They showed that vaporization rate of metals were well enhanced with increasing hydrogen gas mixture ratio under atmospheric pressure. It was proposed "hydrogen plasma-metal reaction" model for the hydrogen assisted high vaporization effect, which is that dissociated hydrogen in the arc plasmas dissolves into molten metals of anode and enhances the vaporization of metal elements when hydrogen atoms are emitted from the molten metals.

The mechanism of the vaporization enhancement by hydrogen in arc plasmas has been still interesting topic. Watanabe et al. have prepared nanoparticles of Si-based inter metallic compounds, and observed the vaporization enhancement of particular metals from metal alloys by hydrogen in arc plasma ${ }^{6}$. Similar tendency was observed in Sn-Ag mixture ${ }^{7)}$. It was shown that this phenomenon can not be explained by only "hydrogen plasma-metal reaction" model.

It is well known that heat flux to the anode is low in argon arcs, and mixtures of argon with helium, hydrogen and nitrogen are used for its enhancement. Thus, arc properties such as maximum

\footnotetext{
*Received: 2008.11 .18

${ }^{* *}$ Member, Institute of Nanotechnology and Material Science, Kurimoto, LTD.

${ }^{* * *}$ Member, Joining and Welding Research Institute Osaka University
}

arc temperature, maximum flow velocity and heat flux density are strongly dependent on ambient gaseous conditions. Recently, Tanaka et al., have investigated the influence of shielding gas composition on arc properties in TIG welding ${ }^{8,9)}$. It was found that the addition of hydrogen to argon increases the arc and anode temperature due to thermal pinch effect ${ }^{8)}$. The changes in arc plasma state may also influence formation of metal fumes or nanoparticles.

In the present study, Fe nanoparticles were prepared by DC arc plasma method. The specific features focused on were the arc constraint (thermal pinch effect) associated with hydrogen gas and its relevance to generation rate and size of the resulting $\mathrm{Fe}$ nanoparticles.

\section{Experimental procedure}

\subsection{Experimental set up}

Fig.1 shows a schematic illustration of experimental set-up. The apparatus mainly consists of the DC power supply, a water-cooled vacuum chamber, a particle collector, a gas

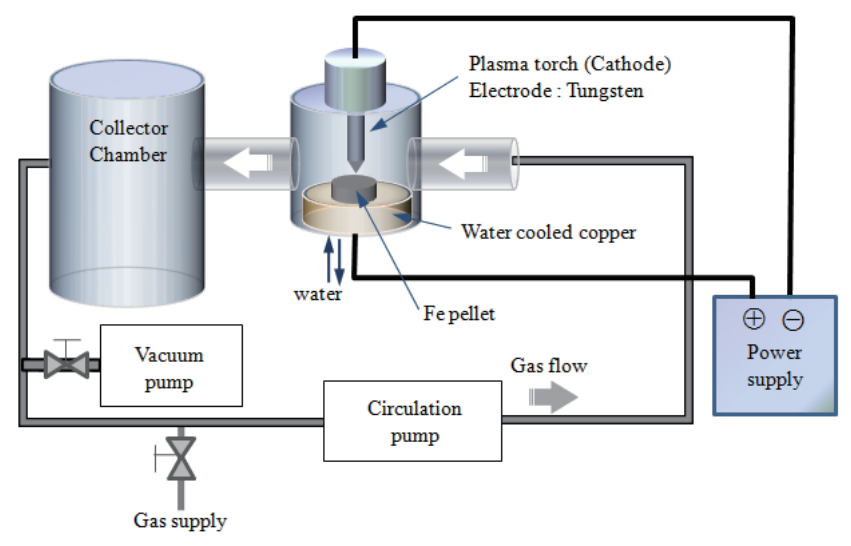

Fig. 1 A schematic illustration of experimental set-up for preparation of $\mathrm{Fe}$ nanoparticles by arc plasma method 
circulator and a vacuum pump. The diameter of tungsten cathode was $8.0 \mathrm{~mm}$ with a $60^{\circ}$ conical tip. The Fe pellet $(99.8 \%)$ was placed on the water-cooled copper anode plate.

Subsequent to achieving a base vacuum of $200 \mathrm{~Pa}, \mathrm{Ar}, \mathrm{H}_{2}$ or Ar- $50 \% \mathrm{H}_{2}$ was introduced. The arc plasma was generated under total pressure of $101 \mathrm{kPa}$ with gas flow rate of $100 \mathrm{l} / \mathrm{min}$. Fe nanoparticles or the generated Fe fume was transported by the gas circulation flow to the collector chamber. In this study, arc current of $150 \mathrm{~A}$ and arc gap of $10 \mathrm{~mm}$ were carefully controlled to be constant during the nanoparticle preparation.

\section{$2.2 \quad$ Analysis}

The weight of the collected $\mathrm{Fe}$ nanoparticles was measured to estimate their generation ratio. The specific surface area of the Fe nanoparticles was measured using a nitrogen gas absorption instrument (micromeritics ASAP 2010, Shimadzu, Japan), based on the BET method, which was used for calculation of the particle size $\left(=6 / \rho S, \rho\right.$ : density, $S$ : specific surface area ${ }^{10)}$. The morphology and the structure were examined by scanning electron microscopy (ERA-8800FE, Elionix, Japan).

The numerical simulation was also conducted for the present plasmas. Using unified models of the arc and the arc electrodes which were developed recently ${ }^{9,11)}$, the maximum arc temperature, the maximum flux flow and the maximum concentration on Fe vapor on the anode were calculated. In all cases, the following parameters were used: an arc current of 150A, an arc length of $10 \mathrm{~mm}$, a $8.0 \mathrm{~mm}$ diameter theoriated tungsten cathode, Fe pellet anode on a water cooled copper, a gas flow rate of $120 \mathrm{~L} / \mathrm{min}$, atmospheric pressure. The details of the calculation are given in Ref.12-13.

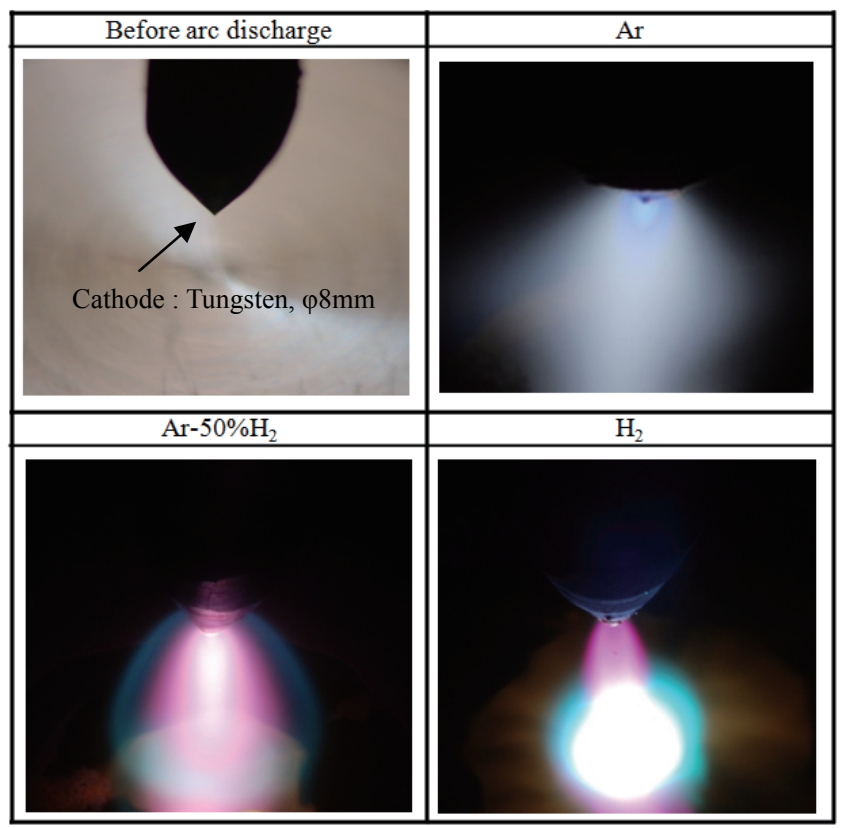

Fig. 2 Photographs of $150 \mathrm{~A}$ arcs in $\mathrm{Ar}, \mathrm{Ar}-50 \% \mathrm{H}_{2}$ and $\mathrm{H}_{2}$.

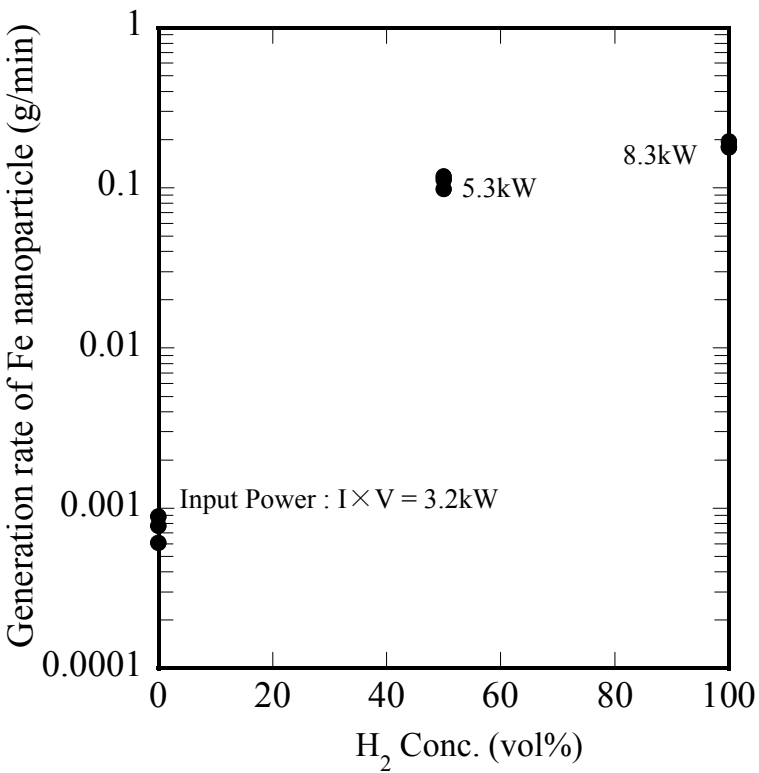

Fig. $3 \mathrm{H}_{2}$ concentration dependence of generation ration of $\mathrm{Fe}$ nanoparticles.

\section{Result and discussion}

Figure 2 shows a set of photographs of $150 \mathrm{~A}$ arcs in Ar, $\operatorname{Ar}-50 \% \mathrm{H}_{2}$ and $\mathrm{H}_{2}$. As can be seen, the arc plasma was significantly constricted by addition of $50 \% \mathrm{H}_{2}$. This is due to the thermal pinch effect ${ }^{8)}$. When only $\mathrm{H}_{2}$ was used, the arc was mostly constricted. The blue colors observed in the $\mathrm{Ar}-50 \% \mathrm{H}_{2}$ and $\mathrm{H}_{2}$ arcs were identified as the ionized $\mathrm{Fe}$ vapor. It is indicated that the hydrogen plays an important role on efficient vaporization of the $\mathrm{Fe}$ anode.

Figure 3 shows the $\mathrm{H}_{2}$ concentration dependence of the generation rate of the $\mathrm{Fe}$ nanoparticles. The significant enhancement of the generation rate was observed for the Ar- $50 \% \mathrm{H}_{2}$ arc, which was increased about two orders of magnitude compared with that of Ar arc. Although $\mathrm{H}_{2}$ arc increased more, the rate of the increase was only slightly higher than that of $\mathrm{Ar}-50 \% \mathrm{H}_{2}$ arc. It is noted that the present dependence is similar to that reported by $\mathrm{Uda}^{14)}$. The generation rate is directly correlated to concentration of $\mathrm{Fe}$ vapor from the anode, and therefore, the vaporization of $\mathrm{Fe}$ was well-enhanced in the Ar- $50 \% \mathrm{H}_{2}$ arc, but more mixing of $\mathrm{H}_{2}, 100 \% \mathrm{H}_{2}$ did not result in further enhancement. The non-linear relationship between $\mathrm{Fe}$ vaporization ratio and $\mathrm{H}_{2}$ concentration in Ar can not be fully explained by the "hydrogen plasma-metal reaction" model ${ }^{4,5)}$.

The input power $(=\mathrm{I} \times \mathrm{V})$ is also shown in Fig.3. It increased with almost proportional to $\mathrm{H}_{2}$ concentration. Since the arc current was fixed to be $150 \mathrm{~A}$, the observed increase of the input 
power was due to the increase of the arc voltage. In arc plasma, input power is consumed mostly for Joule heating, and the heat is transported to anode electrode with very high efficiency. Therefore, in the present study, the increase of $\mathrm{H}_{2}$ concentration must result in the increase of the heat flux density which strongly influences the surface temperature of the anode. The origin of arc properties changed with $\mathrm{H}_{2}$ concentration is also due to the hydrogen induced thermal pinch effect ${ }^{8)}$.

$\mathrm{H}_{2}$ can induce most effectively thermal pinching of the arc. In recent studies performed by Tanaka et al. ${ }^{8,9)}$, the thermal pinch effect strongly depends on specific heat of gas (except for helium). The specific heat of $\mathrm{H}_{2}$ is the most highest among conventionally used gaseous species ${ }^{8)}$, and thereby the arc plasma is mostly constricted under $\mathrm{H}_{2}$ flow. The arc constriction leads to increase of arc voltage under the constant current flow, and the greater ohmic heating gives arise to increase arc temperature, which enhances the heat flux to anode, i.e., metal vaporization from anode. Another interest of the thermal pinch effect is the arc flow velocity, because it also influences formation behavior of $\mathrm{Fe}$ nanoparticles. Decreasing the arc cross-sectional area increases the current density $J$ and the velocity of the arc via magnetic pinch effect, since the pressure gradient is proportional to $J B$, where $B$ is the self-induced magnetic field strength. The enhanced arc flow velocity increases quenching rate of vaporized Fe.

Here, we discuss a possible mechanism of the hydrogen assisted high vaporization effect from point of the thermal pinch effect, with help of our numerical simulation. Table 1 summarizes the calculated maximum arc temperature, maximum arc flow velocity and maximum concentration of $\mathrm{Fe}$ vapor near $\mathrm{Fe}$ anode. As expected, these arc properties are significantly increased with $\mathrm{H}_{2}$ concentration. It is noticeable that the maximum concentration of $\mathrm{Fe}$ vapor in $\mathrm{Ar}-50 \% \mathrm{H}_{2}$ arc is higher about two orders of magnitude compared with that of Ar arc, and is slightly lower than that in $\mathrm{H}_{2}$ arc. These calculated mass ratios are good agreement to those shown in Fig.3.

The maximum concentration of $\mathrm{Fe}$ vapor was calculated by taking account of $\mathrm{Fe}$ vapor pressure which directly relates to the surface temperature of the $\mathrm{Fe}$ anode. In this calculation, the estimated surface temperature of the Fe anode was $2103 \mathrm{~K}$ for $\mathrm{Ar}$ arc.

Table 1 Summary of the calculated arc properties for $150 \mathrm{~A}$ arcs in Ar, Ar- $50 \% \mathrm{H}_{2}, \mathrm{H}_{2}$

\begin{tabular}{|c|c|c|c|}
\hline & $\begin{array}{c}\text { Maximum arc } \\
\text { temperature } \\
(\mathrm{K})\end{array}$ & $\begin{array}{c}\text { Maximum arc } \\
\text { flow velocity } \\
(\mathrm{m} / \mathrm{s})\end{array}$ & $\begin{array}{c}\text { Maximum conc. } \\
\text { of Fe vapor near } \\
\text { anode (mol\%) }\end{array}$ \\
\hline $\mathrm{Ar}$ & 18080 & 184 & 0.02 \\
\hline $\mathrm{Ar}-50 \% \mathrm{H}_{2}$ & 25210 & 796 & 4.07 \\
\hline $\mathrm{H}_{2}$ & 39800 & 4181 & 4.71 \\
\hline
\end{tabular}

This temperature is higher than the melting point of Fe (1808K) and less than the boiling point $(3160 \mathrm{~K})$. On the other hand, the estimated surface temperature was $3027 \mathrm{~K}$ for $\mathrm{Ar}-50 \% \mathrm{H}_{2}$ arc. The surface temperature of the anode heated by $\mathrm{Ar}-50 \% \mathrm{H}_{2}$ arc is very close to the boiling point of $\mathrm{Fe}$, and about $1000 \mathrm{~K}$ larger than that by Ar arc. This is obviously resulted from increase of the heat flux density due to hydrogen induced thermal pinch effect. Therefore, according to the present numerical simulation, vaporization from the $\mathrm{Fe}$ anode could be enhanced by efficiently heated surface of the anode. In case of $100 \% \mathrm{H}_{2}$, since the surface temperature could not be increased more than boiling temperature, it would rather widen the boiled region on the anode, and increase vaporization with depending on the size of boiled region. Thus, the generation ratio of $\mathrm{Fe}$ nanoparticles may be increased slightly than that of $\mathrm{Ar}-50 \% \mathrm{H}_{2}$ arc. The more detail and systematic discussion about the hydrogen assisted high vaporization will be described in a near future, in terms of the thermal pinch effect.

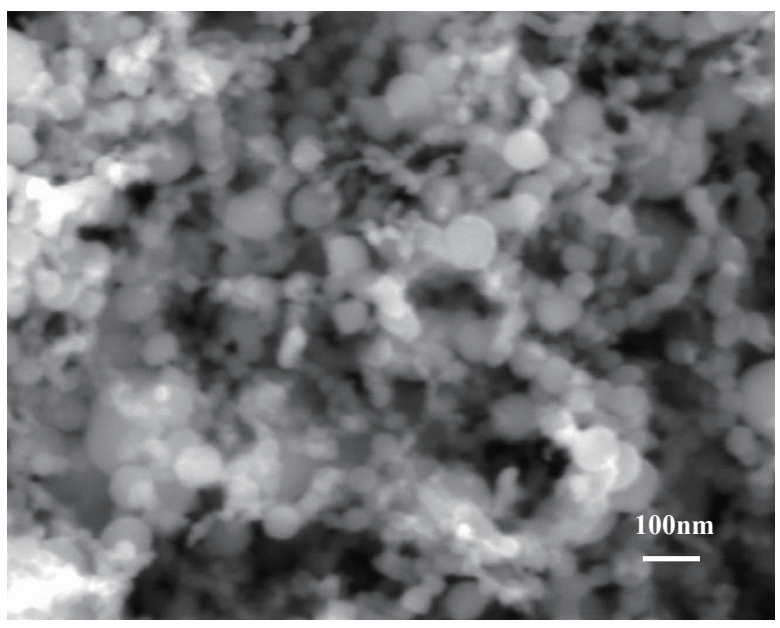

a) $\mathrm{Ar}-50 \% \mathrm{H}_{2}$

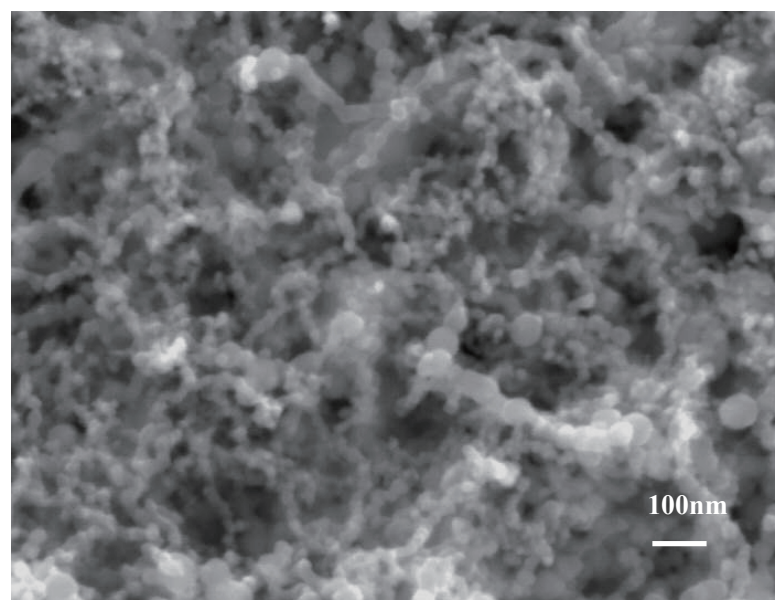

b) $100 \% \mathrm{H}_{2}$

Fig.4 SEM image of Fe nanoparticles produced from a) $\mathrm{Ar}-50 \% \mathrm{H}_{2}$ arc and $\mathrm{H}_{2}$ arc. 
There was no significant difference in the generation ratio for Ar- $50 \% \mathrm{H}_{2}$ and $\mathrm{H}_{2}$ arcs. However, there is a quite difference between them for the arc flow velocity, which would influence the particle size and structures. Figure 4 shows the SEM images of Fe nanoparticles produced from $\mathrm{Ar}-50 \% \mathrm{H}_{2}$ and only $\mathrm{H}_{2}$ arcs. As can be seen, the particles produced from $\mathrm{Ar}-50 \% \mathrm{H}_{2}$ arc is a slightly larger than that of $\mathrm{H}_{2}$ arcs. The particles sizes estimated from average specific surface area for Ar- $50 \% \mathrm{H}_{2}$ and only $\mathrm{H}_{2}$ arcs are $41 \mathrm{~nm}$ and $33 \mathrm{~nm}$, respectively. It can be considered that the higher arc flow velocity in $\mathrm{H}_{2}$ arc would lead to the more nucleation density. On the other hand, the particle size of Fe nanoparticles produced in $\mathrm{Ar}$ arc was 9nm, which was mainly due to the low vaporization of $\mathrm{Fe}$ from the anode. The more detail of nucleation and condensation, i.e., formation behavior of $\mathrm{Fe}$ nanoparticles will be treated in a separate paper.

\section{Conclusions}

Fe nanoparticles were prepared by DC arc plasma method under $\mathrm{Ar}, \mathrm{Ar}-50 \% \mathrm{H}_{2}$ or $\mathrm{H}_{2}$. The thermal pinch effect was induced for $\mathrm{Ar}-50 \% \mathrm{H}_{2}$ or $\mathrm{H}_{2}$ arcs, and $\mathrm{Fe}$ ionized vapor was observed for their arcs. The generation ratio of $\mathrm{Fe}$ nanoparticles was significantly enhanced for $\mathrm{Ar}-50 \% \mathrm{H}_{2}$ or $\mathrm{H}_{2}$ arcs. The numerical simulation of the present arcs revealed that the arc properties of the maximum arc temperature, the maximum arc flow velocity and the maximum are significantly increased with $\mathrm{H}_{2}$ concentration. It is noticeable that the maximum concentration of Fe vapor in $\mathrm{Ar}-50 \% \mathrm{H}_{2}$ arc is higher about two orders of magnitude compared with that of $\mathrm{Ar}$ arc, which are good agreement to the experimental mass ratio of the Fe generation for their arcs. According to our numerical calculations, the enhanced vaporization from the $\mathrm{Fe}$ anode may be due to efficiently heated surface of the anode which relates to hydrogen induced thermal pinch effect. Since the thermal pinch effect also enhanced the arc flow velocity, the particles size of the resulting Fe nanoparticles was influenced even under the similar concentration of $\mathrm{Fe}$ vaporization of $\mathrm{Ar}-50 \% \mathrm{H}_{2}$ and $\mathrm{H}_{2}$ arcs. The present study indicates that control of the thermal pinch effect may be the important factor for producing metallic nanoparticles by arc plasma method.

\section{Acknowledgements}

This work was partially supported by a grand-in-aid for Scientific Research (B) (2008) from the Japan Society for Promotion of Science (JSPS).

\section{Reference}

1) M.De Crescenzi, P.Castrucci, M.Scarselli, M.Diociaiuti, P.S.Chaudhari, C.Balasubramanian, T.M.Bhave and S.V.Bhoraskar: Experimantal imaging of silicon nanotubes, Appl. Phys. Lett., 86 (2005), 231901-1-3.

2) I.Banerjee, Y.B.Khollam, C.Balasubramanian, R.Pasricha, P.P.Bakare, K.R.Patil, A.K.Das and S.V.Bhoraskar: Preparation of $\gamma-\mathrm{Fe}_{2} \mathrm{O}_{3}$ nanoparticles using DC tthermal arc-plasma route, their characterization and magnetic properties, Scripta Mater., 54 (2006), 1235-1240.

3) Z.Cui, Z.Zhang, C.Hao, L.Dong, Z.Meng and L.Yu: Structures and properties of nano-particles prepared by hydrogen arc plasma method, thin solid film, 318 (1998), 76-82.

4) S.Ohno and M.Uda: Generation rate of ultrafine metal particles in "hydrogen plasma-metal" reaction, J. Jpn. Inst. Metals, 48 (1984), 640-646.

5) S.Ohno and M.Uda: Preparation for ultrafine particles of $\mathrm{Fe}-\mathrm{Ni}$, $\mathrm{Fe}-\mathrm{Cu}$ and Fe-Si alloys by "hydrogen plasma-metal" reaction, J. Jpn. Inst. Metals, 53 (1989), 946-952.

6) T.Watanabe, H.Itoh and Y.Ishii: Preparation of ultrafine particles of silicon base intermetallic compound by arc plasma method, thin solid film, 390 (2001), 44-50.

7) M.Tanaka and T.Watanabe: Vaporization mechanism from $\mathrm{Sn}-\mathrm{Ag}$ mixture by $\mathrm{Ar}-\mathrm{H} 2$ arc for nanoparticles preparation, thin solid film, 516 (2008), 6645-6649.

8) M.Tanaka, S.Tashiro, T.Satoh, A.B.Murphy and J.J.Lowke: Influence of shielding gas composition on arc properties in TIG welding, Sci. Technol. Welding and Joining, 13 (2008), 225-231.

9) M.Tanaka and J.J.Lowke: Predictions of weld pool profiles using plasma physics, J. Phys. D: Appl. Phys., 40 (2007), R1-R23.

10) T.T.Kodas and M.H.Smith, AEROSOL PROCESSING OF MATERIALS, Wiley-VCH. (1999), 33.

11) M.Tanaka, H.Terasaki, M.Ushio and J.J.Lowke: A unified numerical modeling of stationary tungsten-inert-gas welding process, Metall. Mater. Trans. A, 33A (2002) 2043-2052.

12) K.Yamamoto, M.tanaka, S.Tashiro, K.Nakata, Y.Yamazaki, E.Ymamoto, K.Suzuki, A.B.Murphy: Numerical simulation of metal vapor behavior in arc plasma, Sur. Coat. Technol., 202 (2008) 5302-5305

13) K.Yamamoto, M.Tanaka, T.Tashiro, K.Nakata, K.Yamazaki, E.Yamamoto, K.Suzuki: Numerical simulation of metal vapor behavior in gas tungsten arc welding, Quarterly J. Japan. Welding Soc., 25-1 (2007) 3-9 (in Japanese).

14) M.Uda, Ultrafine particles, J. Japan Welding Soc., 54 (1985) 318-329. 